\title{
ADVANCING GLOBAL AND REGIONAL REANALYSES
}

Roberto Buizza, Paul Poli, Michel Rixen, Magdalena Alonso-Balmaseda, Michael G. Bosilovich, Stefan Brönnimann, Gilbert P. Compo, Dick P. Dee, Franco Desiato, Marie Doutriaux-Boucher, Masatomo Fujinara, Andrea K. Kaiser-Weiss, Shinya Kobayashi, Zhiquan Liu, Simona Masina, Pierre-Philippe Mathieu, Nick Rayner, Carolin Richter, Sonia I. Seneviratne, Adrian J. Simmons, Jean-Noel Thépaut, Jeffrey D. Auger, Michel Bechtold, Ellen Berntell, Bo Dong, Michal Kozubek, Khaled Sharif, Christopher Thomas, Semjon Schimanke, Andrea Storto, Matthias Tuma, Ilona Välisuo, and Alireza Vaselali

$\mathrm{T}$ his report outlines the structure of and summarizes the recommendations made at the Fifth International Conference on Reanalysis (ICR5), ${ }^{1}$ attended by 259 participants from 37 countries, in Rome, Italy, on 13-17 November 2017. It first summarizes the conference structure. Then, the key recommendations of ICR5 are given for the five main conference topics: production, observations (data rescue and preparation), data assimilation methods, quality assurance of reanalysis, and applications in science, services, and policy making. Finally, five high-level recommendations are proposed for assisting agencies in how best to advance the field of reanalyses, which serves tens of thousands of users, via enhanced research, development, and operations.

ICR5 STRUCTURE. ICR5 was the fifth in a series of international conferences for researchers, developers, and producers of reanalyses. The previous event, ICR4, took place in Maryland, during 2012, and attracted 244 participants (www.wcrp-climate.org /ICR4). The sharp increase in the number of countries represented, from 26 in 2012 to 37 in 2017, shows that reanalyses are now used by a wider range of

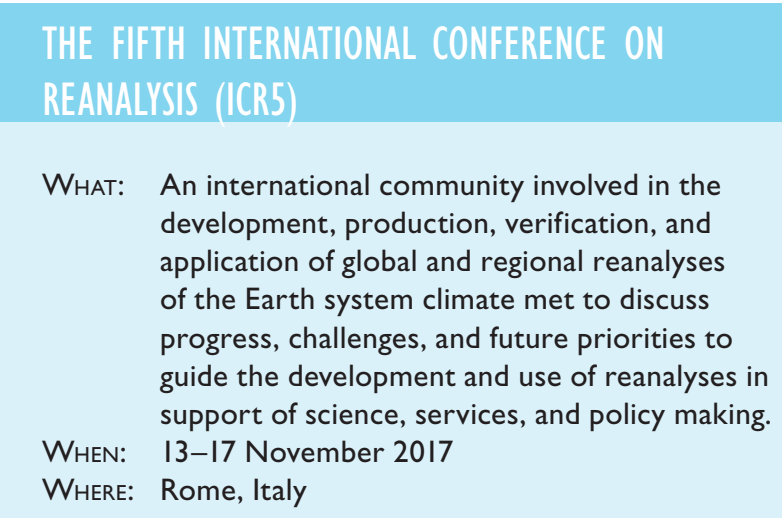

stakeholders, including not only the scientific community, but also public organizations and the private sector in need of long-range time series of climate information. Applications are not limited to research and include climate services and policy making.

This conference was timely as several events have taken center stage since ICR4 in the climate domain, such as the 2015 Paris Agreement on climate, reached at the 21st Conference of the Parties (COP21) to the United Nations Framework Convention on Climate

\footnotetext{
${ }^{1}$ ICR5 was co-organized by the European Centre for Medium-Range Weather Forecasts (ECMWF), the European Union's (UN) Copernicus Climate Change Service operated by ECMWF on behalf of the European Commission (ECMWF/C3S), and the WCRP. The ICR5 Scientific Organizing Committee (ICR5-SOC) was cochaired by Roberto Buizza (ECMWF) and Paul Poli (Météo-France), and included 20 experts from 17 world-renowned institutions (coauthors of this article). Session chairs and rapporteurs, including early career scientists, also contributed to this article.
} 
Change (UNFCCC). The ICR5 participants discussed the merits, limits, and challenges of reanalyses for monitoring climate variability and change, for supporting policy makers in the development of adaptation and mitigation measures, and for providing information complementary to that from other climate sources.

ICR5's presentations, oral and posters, ${ }^{2}$ were organized around five topics:

1) status and plans for reanalysis production (production, inclusive of all Earth system thematic areas-atmosphere, land, ocean, and cryosphere),

2) observations for reanalysis (preparation, organization in large archives, data rescue, and reanalysis feedback),

3) methods for reanalysis (data assimilation, Earth system coupling, uncertainty estimation, and challenges specific to regional reanalyses),

4) evaluation of reanalyses (comparisons with observations, other types of analysis and models, intercomparisons, and diagnostics), and

5) applications of reanalyses (generation of time series of essential climate variables for climate monitoring, climate services, and industry applications).

The call for abstracts reached over 30,000 users through various channels, and 357 unique abstracts were received from 53 countries. However, owing to limited travel support, participants from 16 countries were not able to attend in person. For this reason, the conference organizers welcomed a sponsor contribution that supported live video streaming of the conference presentations and debates, of which recordings are available (www.youtube.com/channel /UCdK5sfMQcJ64q8AGR_7-ZRw/videos). The resulting conference program included a media briefing, 66 oral presentations, one panel discussion, a plenary discussion, and three poster sessions, where a total of 201 posters were presented.

The organizers took gender balance into consideration when selecting the invited presentations (four keynotes, plus one invited talk per topic), with four invited women speakers and five invited male speakers. In total, 66 talks were given and the ratio of female to male speakers was 18:48, or 27\%:73\%. Each session was introduced by an invited talk, given by Hisashi Nakamura, Nick Rayner, Patrick Heimbach, Masatomo Fujiwara, and Karina Von Schuckmann, which gave a gender ratio of 2:3 of women to men.

The chairs and rapporteurs of the five topics reported back to the plenary discussion the findings

\footnotetext{
${ }^{2}$ All presentations and posters can be accessed from the ICR5 website (http://climate.copernicus.eu/events/5th -international-conference-reanalysis).
}

AfFiliations: BuizZA ANd Alonso-Balmaseda-European Centre for Medium-Range Weather Forecasts, Reading, United Kingdom; Poll-Météo-France, Brest, France; Rixen AND Tuma-World Climate Research Programme, World Meteorological Organization, Geneva, Switzerland; BosıLOVICH-Global Modeling and Assimilation Office, NASA GSFC, Greenbelt, Maryland; BRÖNNIMANN—Institute of Geography, University of Bern, Bern, Switzerland; CoMPO-University of Colorado Boulder, and Cooperative Institute for Research in Environmental Sciences, and NOAA/ESRL/Physical Sciences Division, Boulder, Colorado; DeE, SIMmons, AND THÉPAUT-European Centre for Medium-Range Weather Forecasts, and Copernicus Climate Change Service (C3S), European Commission, Reading, United Kingdom; DESIATO-Istituto Superiore per la Protezione e la Ricerca Ambientale, Rome, Italy; DOUTRIAUX-BOUCHER-European Organisation for the Exploitation of Meteorological Satellites, Darmstadt, Germany; FujIWARA—Faculty of Environmental Earth Science, Hokkaido University, Hokkaidō, Japan; KAISER-WEISS—Deutscher Wetterdienst, Offenbach, Germany; KOBAYASHI-Japan Meteorological Agency, Tokyo, Japan; LıU—National Center for Atmospheric Research, Boulder, Colorado; MASINA-Fondazione Centro Euro-Mediterraneo sui Cambiamenti Climatici (CMCC), and Istituto Nazionale di Geofisica e Vulcanologia, Bologna, Italy; MATHIEU-European Space Agency, Frascati, Italy; Rayner-Met Office, Exeter, United Kingdom;
RICHTER-Global Climate Observing System, World Meteorological Organization, Geneva, Switzerland; Seneviratne-Eidgenössische Technische Hochschule Zürich (ETH Zürich), Zurich, Switzerland; AUGER-Climate Change Institute, University of Maine, Orono, Maine; BECHTOLD—Department of Earth and Environmental Sciences, and Department of Computer Science, Katholieke Universiteit Leuven, Leuven, Belgium; BERNTELL-Stockholm University, Stockholm, Sweden; Dong AND THOMAs-Department of Meteorology, University of Reading, Reading, United Kingdom; KozUBeK-Institute of Atmospheric Physics, Academy of Sciences of the Czech Republic, Prague, Czech Republic; SHARIF-ArabiaWeather Inc., Amman, Jordan; SCHIMANKE-Swedish Meteorological and Hydrological Institute, Norrköping, Sweden; STORTO_CMCC, Bologna, Italy; VÄLISUOFinnish Meteorological Institute, Helsinki, Finland; VASELALI-Beta Group Consulting Engineers, Tehran, Iran CORRESPONDING AUTHOR: Roberto Buizza, roberto.buizza@ecmwf.int

DOI:10.1I75/BAMS-D-17-0312.I

In final form 26 February 2018

(C)2018 American Meteorological Society

For information regarding reuse of this content and general copyright information, consult the AMS Copyright Policy. 
and recommendations of their sessions, which are summarized hereafter.

ICR5 KEY RECOMMENDATIONS. During the ICR5 plenary discussions, it was agreed to share with the scientific communities a set of key recommendations, which have been organized into the five main topics discussed during the conference.

Status and plans for reanalysis production. Since ICR4 in 2012, an increasing number of reanalyses have been produced: global and regional, uncoupled and coupled. There has been a continued effort to improve their quality (accuracy) and to represent more and more Earth system components (atmosphere, ocean, land, cryosphere, aerosols, biogeochemistry, and hydrosphere). Resolution has been increased, and the time spanned by various reanalyses has been extended to cover the entire twentieth century and beyond. Ensemble methods have been developed to address uncertainty estimation. Discontinuities owing to observing system variations over the past decades remain a significant issue in the production of climate (long time series) reanalyses. In this area of work, the following recommendations are made:

1) Given the extensive use of reanalyses at climate and weather time scales, and especially as centers move toward more sophisticated coupled Earth system reanalyses, ICR5 recommends centers generate families (or extensions) of reanalyses to support climate studies and monitoring using different observing system configurations spanning the observational record (longer climate timescale reanalyses alongside reanalyses spanning the period of the modern observing system).

2) Improved communication between producers and users is required to guide the latter in their application and interpretation.

3) Short-term latency products, in addition to the full reanalysis, would increase the reanalyses' potential applications.

Observations for reanalysis. Observations are needed to monitor our changing climate and are a crucial reanalysis ingredient: what is assimilated and its quality is key to the success of the reanalysis. Work on observations for reanalysis requires a sustained, well-supported effort involving cooperation with reanalysis producers. Existing initiatives and programs need to be strengthened and enhanced to guarantee that so-called anchor series, a subset of observations required for reanalyses and key to reduce

\section{WHAT ARE REANALYSES?}

- Reanalyses are digital datasets that represent the evolution of the Earth's climate system. They cover the diversity and complexity of the Earth's climate system: multicomponents (e.g., atmosphere, ocean, land, ice), multivariables (e.g., temperature, humidity, wind, ozone), and multidimensions (e.g., subdaily to seasonal scales, regional to global scales, ocean subsurface depths to the surface where we live to the upper atmosphere), with a growing role for the human factor (anthropogenic), without which one cannot understand or reproduce some recent climate evolutions (such as ozone depletion and the increase in greenhouse gases).

- Reanalyses bring together the full history of observations and our growing computing power, and blend all this using the latest science (including advances in mathematics, physics, and information technology). Registered user counts at several institutions exceed tens of thousands.

- Reanalyses enable the placement of present weather events within a climate context and allow us to revisit the past. Reanalysis is, for example, a key contribution to the Global Framework for Climate Services (GFCS), allowing monitoring of the Earth's climate even in places where direct observations are sparse. Reanalysis data have been used for continuing the development and improvement of weather forecasting, climate services, and climate change monitoring.

- Reanalyses are coordinated internationally under the auspices of the WCRP, which is cosponsored by the World Meteorological Organization (WMO), the International Council for Science (ICSU), and the Intergovernmental Oceanographic Commission (IOC) of the United Nations Educational, Scientific and Cultural Organisation (UNESCO).

uncertainties, are more consistent. Key observations to be considered as anchor series are sea surface temperature, sea ice concentration and thickness, Argo profiling float data, global positioning system (GPS) radio occultation (GPSRO) data, radiosonde, surface pressure, scatterometer wind, and soil moisture. Sustained production of carefully curated underlying key datasets is essential to avoiding discontinuities that affect downstream services. In this area of work, the following recommendations are made:

1) More research and operational funding should be made available to support the tools, methodologies, and essential steps of data preparation: rescue, assembly, reprocessing, recalibration, bias correction, quality control, homogenization, 
uncertainty quantification, use in reanalysis, and feedback analysis.

2) Funding of essential datasets needs to be increased and sustained. Funding for their maintenance and improvements has been cut to below a sustainable level.

3) In situ and satellite observing systems that have been proven to serve as critical references (also referred to as anchors) for reanalysis need to be preserved and extended in time. These include measurements of sea surface temperature, sea ice concentration and thickness, atmospheric surface pressure, and soil moisture, as well as measurements by radiosondes, ocean profiling floats and the moored buoy array, GPS radio occultation, and scatterometers from satellites.

4) Data rescue of historical observations and reprocessing of observations (e.g., satellite) to improve their quality and utility needs to be continued.

5) The sea ice and meteorological observations for the Southern Ocean, recently discovered in various archives for the late nineteenth and early twentieth centuries, should be digitized to improve our understanding and representation of this key region.

Methods for reanalysis. Reanalysis methods have been improving considerably in the past five years. Ensembles are increasingly being used, either exclusively or in hybrid systems. Other techniques, such as particle filters/smoothers, are being tested. Coupled approaches (e.g., ocean, land, cryosphere, and atmosphere) have been shown to be promising and yield benefits. Historical reanalyses going back to the nineteenth century and beyond have been created. Improvements in the methods, to include more robust uncertainty quantification associated with observations, models, and data assimilation, are needed. In this area of work, the following recommendations are made:

1) Reanalysis is not a research priority for most national and international programs. However, reanalyses are among the most used datasets. As a result of this lack of priority, most of the data assimilation development adopted for reanalysis currently takes place where resources are available, at numerical weather prediction (NWP) centers. They predominantly focus on their usual (i.e., near-real time) requirements, rather than the use of historical observations, to reconstruct a climate record. These centers and the community at large need to incorporate clearly articulated requirements for climate reanalysis in the development pathways for data assimilation. Systems do not work "off the shelf" to reanalyze observations 10, 20, or 100 years earlier.

2) There is a gap in research funding for designing data assimilation methods specifically targeting climate reanalysis. Those developments are required and need to be adequately resourced and prioritized.

3) The EU Copernicus project has brought substantial resources to improving data access, understanding end-user requirements, enhancing observational databases, carrying out global and European regional reanalyses, and providing operational services based on reanalysis. However, there is no similar system or mechanism for supporting the underlying research needed to improve and sustain these services and some of the downstream regional reanalysis. This also appears to be the case across non-EU countries.

Evaluation of reanalyses. The evaluation of new, stateof-the-art reanalyses has shown improvement in the representation of several different processes, among them precipitation and soil moisture. Comprehensive intercomparisons have been carried out (e.g., projects such as an atmospheric reanalysis intercomparison project that focuses on the stratosphere-troposphere interaction and an ocean reanalysis intercomparison project that focuses on the ocean). Recent evaluation and diagnostic activities have found that reanalyses capture changes in climate extremes in addition to mean changes. There are substantial new developments in regional reanalyses, for example, for the Arctic region and for Europe and other continents. These products have been shown to be regionally of higher accuracy than global equivalent ones. With respect to the evaluation of reanalyses, the following recommendations are made:

1) Diagnostic and evaluation activities that look at the mean climate, variability, and extremes of coupled atmosphere-ocean-land Earth system reanalyses are strongly encouraged, since they are of high relevance for climate applications.

2) Increased assimilation of land information (e.g., microwave soil moisture estimates, terrestrial water storage estimates from satellite gravity missions, skin temperature, and land precipitation) is encouraged to improve the representation of temperature extremes on land.

3) More interaction between research groups working on regional and global reanalyses is encouraged. It is recommended that regional 
reanalyses be integrated into international activities on the intercomparison of reanalysis products.

Applications of reanalyses. There are a growing number of applications, in a variety of sectors (e.g., wind energy and hydrology). There is a common request to have easier data access, and more clear guidance (e.g., on the estimation of reanalyses' uncertainty). Desire for higher resolution and accuracy for surface variables is a driving demand for regional reanalysis and various downscaling techniques. With respect to applications of reanalyses, the following recommendations are made:

1) The priority given to reanalysis activity needs to be raised to the level of its impact. Reanalyses are crucial for climate monitoring, to initialize weather reforecasts used to generate operational weather products [see, e.g., the ECMWF and the National Centers for Environmental Prediction (NCEP) experiences] and climate predictions, and to verify their accuracy across long time records. They represent a fundamental reference to which those models can be compared in order to evaluate the merits and skill of different prediction systems across a wide range of complementary metrics.

2) Continuous production is recommended to achieve higher resolution, faster access, longer time series, better quality, and couplings with the components of the Earth system (atmosphere, ocean, land, ice, atmospheric chemistry, and the biosphere and hydrosphere). Due consideration of the anthropogenic influence may need more attention in future reanalyses (e.g., irrigation, land-use change, and urbanization).

3) Better and more "actionable" uncertainty characterization is required: users request simple measures of the reanalyses' uncertainty.

4) The input needed by applications is still dependent on the uncertainty and accuracy of observations and data assimilation techniques. There are still many observations missing in data-sparse regions, or from historic periods, and a need for less constrained parameters, such as SST, sea ice, deep ocean information, and soil moisture.

5) Intercomparison and evaluation activities need to continue for global and regional reanalyses, in order to benchmark each dataset.

6) The user community is asking for reanalyses of higher resolution, faster access, longer time series, and better quality. A special interest was noted for coastal and urban applications.
7) Enhanced communication between producers and users is required. Platforms for exchanging information-like the website Reanalyses.org or the National Center for Atmospheric Research's (NCAR) Climate Data Guide-should continue to promote such discussion. In addition, events like ICR5 are very successful in facilitating the exchange of information.

\section{MESSAGE TO MANAGING AGENCIES.}

ICR5 clearly illustrated the leading role of various agencies in this field, built throughout the years. For example, the establishment of the Copernicus program and its various services, as well as sustained support from ECMWF, the National Aeronautics and Space Administration (NASA), the National Oceanic and Atmospheric Administration (NOAA), Japan Meteorological Agency (JMA), the European Space Agency (ESA), the European Organisation for the Exploitation of Meteorological Satellites (EUMETSAT), the U.S. Department of Energy (DoE), the European Commission (EC), and several other institutions, have helped to consolidate progress. The following are five distilled recommendations for agencies and supporting institutions to enable further progress in this area:

1) Reanalysis production-As production centers move toward coupled Earth system reanalyses, they (and their users) should embrace the notion of families of products designed to support a variety of research and applications spanning multiple decades to centuries. Reduced latency of reanalysis data products (ideally in real time, with related requirements on the latency of climate observations) should be aimed for, since it increases reanalyses' potential for applications.

2) Observations for reanalysis-Supporting the rescue, reprocessing, recalibration, correction, quality control, and use of observations for reanalysis is key to enhancing the scope and range of reanalyses, by allowing datasets to be extended back in time with high-quality observations. Integrated datasets for marine and land surface, such as the International Comprehensive Ocean-Atmosphere Data Set (ICOADS), are essential. Looking ahead to the continuation of climate time series, there is an urgent need to strengthen and enhance the Global Climate Observing System (GCOS) and the Global Ocean Observing System (GOOS), which includes support for implementing critical in situ and satellite measurements. 
3) Methods for reanalysis-Although the conference reported on new ideas (smoothers), there is a general gap in research funding to design data assimilation and coupling methods across the Earth system specifically designed for reanalysis.

4) Evaluation of reanalyses-Diagnostic and evaluation activities that look at the mean climate, variability, and extremes of globally coupled atmosphere-ocean-land Earth system reanalyses should be promoted, taking stock of successful examples of ocean (and atmosphere) reanalysis intercomparison projects. Increased interaction between research groups working on regional and global reanalyses would be highly beneficial. The World Climate Research Programme (WCRP)
Task Team for the Intercomparison of Reanalyses (TIRA; https://reanalyses.org/wcrp-task-team -intercomparison-reanalyses-tira) is charged with developing plans for promoting and coordinating such activities with the community and platforms, such as Reanalyses.org and the NCAR Climate Data Guide.

5) Applications of reanalyses-Synergies between reanalyses' producers and users must be increased. There is a need for better and more applicable uncertainty characterization. The proper quantification and communication of the quality of reanalyses must be promoted and would broaden their usage in operational services and policy making. 